Research Article

\title{
Expression and Clinical Significance of Cancer Stem Cell Markers CD24, CD44, and CD133 in Pancreatic Ductal Adenocarcinoma and Chronic Pancreatitis
}

\author{
L. Durko, ${ }^{1}$ W. Wlodarski, ${ }^{1}$ O. Stasikowska-Kanicka, ${ }^{2}$ M. Wagrowska-Danilewicz, ${ }^{2}$ \\ M. Danilewicz, ${ }^{2}$ P. Hogendorf, ${ }^{3}$ J. Strzelczyk, $^{3}$ and E. Malecka-Panas ${ }^{1}$ \\ ${ }^{1}$ Department of Digestive Tract Diseases, Medical University of Lodz, Lodz, Poland \\ ${ }^{2}$ Department of Pathomorphology, Medical University of Lodz, Lodz, Poland \\ ${ }^{3}$ Department of General and Transplant Surgery, Medical University of Lodz, Lodz, Poland \\ Correspondence should be addressed to L. Durko; lukasz.durko@umed.lodz.pl
}

Received 31 January 2017; Revised 11 April 2017; Accepted 24 April 2017; Published 4 June 2017

Academic Editor: Stamatios E. Theocharis

Copyright ( 2017 L. Durko et al. This is an open access article distributed under the Creative Commons Attribution License, which permits unrestricted use, distribution, and reproduction in any medium, provided the original work is properly cited.

\begin{abstract}
Cancer stem cells (CSC) play an important role in pancreatic carcinogenesis and prognosis. The study aimed at examining the expression of CD24, CD44, and CD133 in human PDAC and CP in order to evaluate its clinicopathological correlations and the clinical significance. Surgical specimens from 23 patients with PDAC and 15 patients with chronic pancreatitis after pancreatic resection were stained with $\mathrm{CD} 24, \mathrm{CD} 44$, and CD133 antibodies. The intensity of staining was scored from 0 (negative) to 3 (strongly positive). Results. Mean CD24 staining score in PDAC was $1.38 \pm 0.76$ and was significantly higher than that in CP: $0.70 \pm 0.53(p<0.01)$; CD44 score in PDAC was $2.23 \pm 0.42$ and was significantly higher than that in CP: $1.87 \pm 0.55(p<0.05)$; CD133 score $0.93 \pm 0.58$ was not different from CP: $0.71 \pm 0.43(p>0.05)$. CD44 immunoreactivity was significantly higher $(p<0.05)$ in pT1 and pT2 patients together as regards pT3: $2.45 \pm 0.37$ versus $2.06 \pm 0.38$ as well as in N0 patients compared to N1 patients: $2.5 \pm 0.38$ versus $2.04 \pm 0.34$. Conclusions. CD24 and CD44 are upregulated in human pancreatic cancer compared to chronic pancreatitis. CD44 immunoreactivity decreases with the tumor advancement and may represent the negative PDAC prognostic factor. Each CSC marker was differently related to PDAC advancement. CD133 may lack clinical significance in PDAC.
\end{abstract}

\section{Introduction}

The incidence of and mortality associated with pancreatic ductal adenocarcinoma (PDAC) have increased during the last decade. It is expected that by the year 2030, pancreatic cancer will become the second most prevalent cause of cancer-related deaths [1]. One of the reasons associated with high malignancy of PDAC is the presence of a subpopulation of chemoresistant, self-renewable, and multipotent cells in the bulk of tumor termed cancer stem cells (CSC). These cells are believed to be responsible for tumor initiation, rapid growth, resistance to therapy, recurrence, and metastasis. CSC have been found in several types of neoplasms [2-5] including pancreatic cancer
[6, 7]. In 2007, $\mathrm{Li}$ and colleagues identified a highly tumorigenic subpopulation of cells in human PDAC. Transplantation of this subpopulation of cells to immunocompromised mice led to initiation and systemic spread of pancreatic cancer in experimental animals. Interestingly, these cells express surface markers CD44, CD24, and epithelial-specific antigen (ESA). Such phenotype was present in $0.2-0.8 \%$ of PDAC cells and corresponded to their 100 -fold increased tumorigenic potential compared with nontumorogenic cancer cells [6].

Hermann et al. demonstrated that pancreatic CSC show high expression of CD133 (prominin-1) [7]. CD133 is a transmembrane glycoprotein responsible for regulation of multiple cell signalling pathways, including Akt/PKB, 
Bcl-2, Ras, and its downstream effectors such as ERK, JNK, PI3K, and p38K [8].

CD24, a small cell surface protein anchored by glycosylphosphatidylinositol, is heavily glucosylated and is involved in cell-cell and cell-matrix interactions. CD24 tends to be expressed at higher levels in progenitor cells and metabolically active cells and to a lesser extent in well-differentiated cells. The function of CD24 is unclear for most types of cells [9].

CD44 is the transmembrane glycoprotein that can act as a receptor for extracellular matrices as hyaluronic acid and is the downstream target of the $\mathrm{Wnt} / \beta$-catenin pathway. CD44 expression was related to a more aggressive course and presence of metastases in PDAC $[10,11]$.

The CSC presenting self-renewable properties involve also other signaling pathways such as Notch and Hedgehog. Overexpression of Notch-1 results in increased cell proliferation, migration, and invasion of cancer cell lines. Additionally, high levels of CD44 were observed in PDAC with increased Notch-1 expression [12].

Pancreatic CSC also influence the Hedgehog signaling pathway. It has been shown that a ninefold increase in Sonic hedgehog mRNA levels were present in the CD44 and CD24 PDAC positive cells compared to the pancreatic cancer cells with low or absent expression of these biomarkers [6]. Interestingly, Rodova et al. showed that blocking hedgehog pathway by sulforane inhibits the proliferation of CSC [13].

Another mechanism involved in CSC function involves mTOR pathway. The mTOR inhibition by rapamycin in in vitro studies resulted in decreased viability of CD133 PDAC cells and their reduced self-renewal properties [14].

Many authors have proven that isolated pancreatic CSC are more chemoresistant compared to non-CSC cancer cells [15-17]. Therefore, elimination of CSC in PDAC could improve the treatment results and lead to a better prognosis in patients affected by this disease. Multiple new strategies to target stem cells are being investigated [18-20]. The more precise identification of pancreatic CSC may help to better understand the biology of PDAC and allow for successful development of more accurate and targeted therapies.

Chronic pancreatitis (CP) is associated with increased risk of PDAC [21]. Nevertheless, the nature of the transition between $\mathrm{CP}$ and PDAC is poorly understood. Fibrosis is a hallmark of human PDAC and CP. The elevated serum concentration of cytokines involved in inflammatory processes such as IL-6, TGF $\beta$, and TNF $\alpha$ are observed both in PDAC and CP $[22,23]$. It has been suggested that stellate cells represent pancreas resident CSC in the course of pancreatic inflammation and can activate the pathways required for malignant transformation of epithelium, promotion of migration, and formation of distant metastases [24].

So far, there is very little data regarding the comparison of the expression of CD24, CD44, and CD133 in PDAC and chronic pancreatitis (CP). There is no sufficient information regarding the role of these biomarkers in CP. However, such comparative analysis might lead to better characterization of stem cell function in both diseases.

\section{Aim of the Study}

The study aimed at examining the expression of CD24, CD44, and CD133 in human PDAC and CP in order to evaluate their clinicopathological correlations and their clinical significance.

\section{Material}

Surgical specimens derived from 23 pancreatic cancer patients (10 women and 13 men, aged 40-75; mean 56.09 \pm 9.38 ) and 15 patients with CP ( 3 women and 12 men, aged $36-65$; mean $48.86 \pm 10.57$ ) were subjected to pathology and immunohistochemistry studies. $\mathrm{CP}$ and PDAC diagnosis was based on medical history and imaging studies (abdominal ultrasound, EUS, and computed tomography) and confirmed with pathology. Qualification for surgical intervention among $\mathrm{CP}$ patients was based on the following indications: detection of tumor in imaging techniques or severe pain unresponsive to medical treatment. The following criteria were used to qualify for resection of PDAC: lack of distant metastases, no infiltration of major blood vessels, and/or lack of invasion of the upper part of the portal vein or the lower part of the superior mesenteric artery that allows for surgical reconstruction of the vessel.

The differentiation grade of PDAC was G1 in 6 cases, G2 in 14 patients, and G3 in 3 individuals. Classification of patients according to the TNM revealed the following stages: pT1 in 3 cases, pT2 in 7 patients, and pT3 in 13 individuals. Lymph node involvement was as follows: N0 in 7 patients, N1a in 5 cases, N1b in 8 patients, and Nx in 3 individuals. Generally, no distant metastases were detected other than small liver metastases $(n=2)$ and splenic vein involvement $(n=2)$.

Parameters related to patient demographics, clinical data, grade, and stage of the disease (according to TNM scale) were correlated with CD24, CD44, and CD133 expression. Patients' survival was calculated from the time of diagnosis until death.

\section{Methods}

Tissue expression of CD133, CD24, and CD44 were assessed with the use of Miltenyi Biotec (Germany), Becton Dickinson (New Jersey, USA), and Dako (Denmark) antibodies (resp.). The intensity and extent of CD24, CD44, and CD133 staining were taken into consideration and scored on a scale of $0-3$, in which 0 referred to negative, 1 to weakly positive, 2 to moderately positive, and 3 to strongly positive staining. Seven to ten high-power microscopic fields were evaluated. Staining of KI-67+ cells was evaluated using the computer image analysis system consisting of PC computer equipped with a Pentagram graphic tablet, Indeo Fast card (frame grabber, true color, real time; Taiwan), and Panasonic color TV camera coupled with Carl Zeiss microscope (Germany). This system was programmed by Multiskan 8.08 software, Computer Scanning Systems, Poland. Ki-67 labeling index (LI) was estimated counting 100 cells in ten monitor fields $\left(0.029 \mathrm{~mm}^{2}\right.$ each), marking immune-positive cells, so in each case, at least 1000 cells were analyzed. The survival 


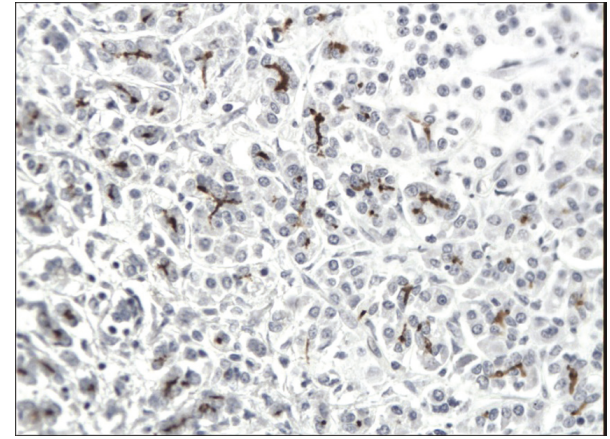

Figure 1: Almost negative immunoexpression of CD24 in epithelial cells in chronic pancreatitis. A weak immunoexpression in stromal cells is seen (magn. 200x).

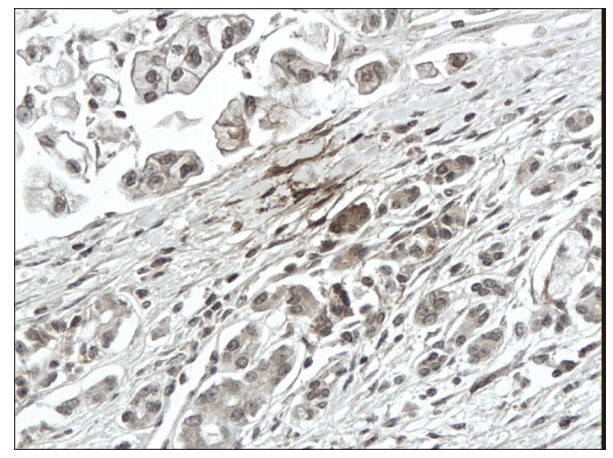

FIGURE 2: Focal cytoplasmic and membranous immunoexpression of $\mathrm{CD} 24$ in pancreatic cancer. A weak immunoexpression of stromal cells is also seen (magn. 200x).

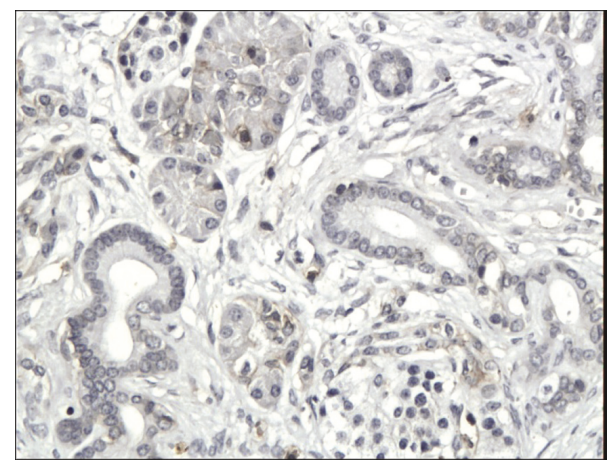

FIGURE 3: Weak immunoexpression of CD44 in chronic pancreatitis (magn. 200x).

probability of PDAC patients depending on the expression of $\mathrm{CD} 24, \mathrm{CD} 44$, and CD133 was estimated by KaplanMeier analysis.

\section{Results}

The immunoexpressions of CD24, CD44, and CD133 were both membranous and cytoplasmic. In PDAC, CD24 immunoreactivity was found in 19 (82.6\%) patients, CD133 in 21 (91.3\%) and CD44, as well as, Ki-67 in all of examined individuals. In $\mathrm{CP}, \mathrm{CD} 24$ staining was found in $11(73.3 \%)$

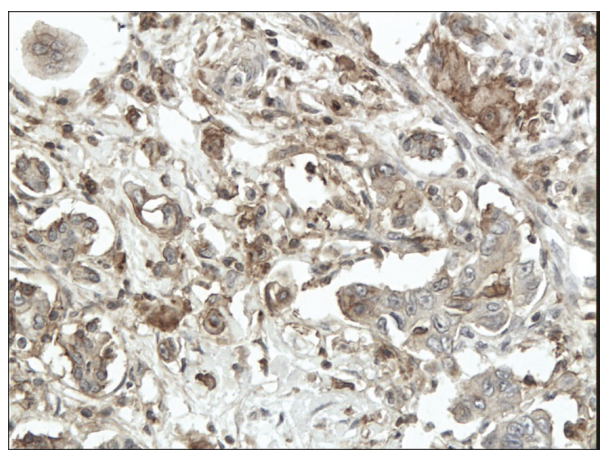

FIGURE 4: Intense, predominantly cytoplasmic immunoexpression of CD44 in pancreatic cancer (magn. 200x).

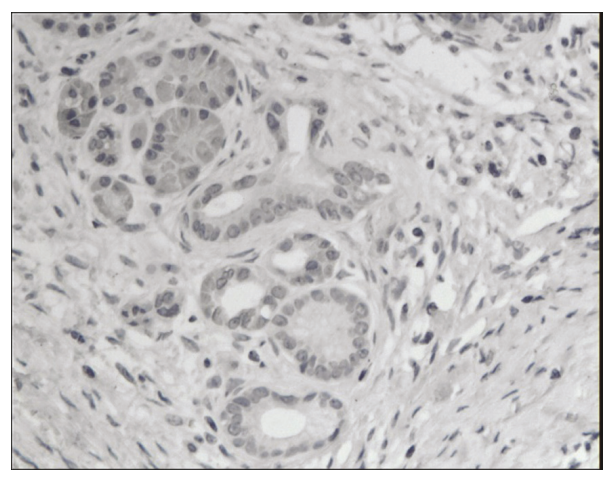

FIGURE 5: Weak, mainly membranous immunoexpression of CD133 in chronic pancreatitis (magn. 200x).

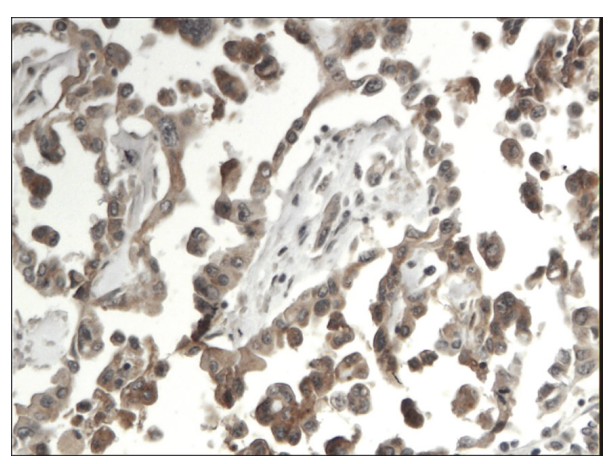

FIgure 6: Moderate immunoexpression of CD133 in patients with pancreatic cancer (magn. 200x).

patients, CD133 in $13(86.6 \%)$ and CD44 as well as Ki-67 in all of them. Mean CD24 staining score in PDAC was $1.38 \pm 0.76$ and was significantly higher than that in $\mathrm{CP}$ $0.70 \pm 0.53(p<0.01)$. In our study, CD44 score in PDAC was $2.23 \pm 0.42$ and was again significantly higher than that in CP $1.87 \pm 0.55(p<0.05)$. CD133 score observed in patients with pancreatic cancer was of $0.93 \pm 0.58$ and was not different from the one detected in CP patients 0.71 \pm 0.43 ( $p>0.05)$. Both in PDAC and CP individuals, immunohistochemistry demonstrated the lowest expression of CD133 and the highest of CD44 (Figures 1-7). CD44 


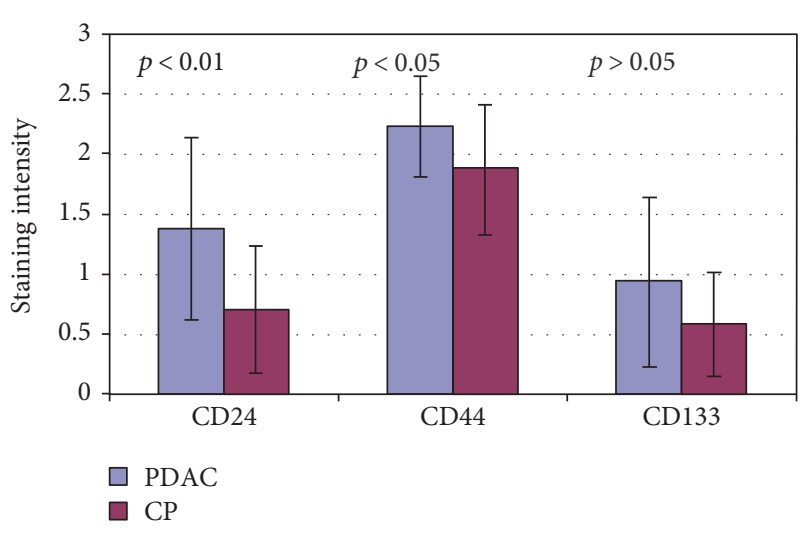

Figure 7: Mean CD24, CD44, and CD133 intensity of staining in PDAC and CP.

immunoreactivity was significantly higher $(p<0.05)$ in pT1 and pT2 patients considered together as regards pT3:2.45 \pm 0.37 versus $2.06 \pm 0.38$, as well as in N0 patients compared to those individuals, who presented with involvement of lymph nodes: $2.5 \pm 0.38$ versus $2.04 \pm 0.34$ (Figure 8). The immunoreactivity of Ki-67 LI in chronic pancreatitis was higher in PDAC comparing to CP: 54.09 versus $1.56(p<0.05)$. We found a negative correlation between the CD24 expression and Ki-67 LI in PDAC $(r=-0.772$; $p<0.05$ ) (Figure 9). No significant correlation has been found between CD44 and CD133 expression and Ki-67 LI in PDAC. Additionally, there was no correlation between CD24, CD44, and CD133 expression and patients' age and tumor grade. Moreover, Kaplan-Meier analysis showed no significant relation of survival of PDAC patients and the expression of CD24, CD44, and CD133 (Figures 10-12).

\section{Discussion}

Data regarding the expression of CSC markers, especially those of CD133, CD44, and CD24 in patients with PDAC and/or CP is scarce. Our study showed that the expression of CD133, CD24, and CD44 is present in both CP and PDAC. In addition, the expression of CD24 and CD44 was higher in malignant tumors compared to that in an inflamed pancreatic tissue. No such differences were seen in CD133 expression.

Immervoll et al. analyzed the expression of CD44 and CD133 in surgical samples of PDAC, noncarcinoid pancreatic tumors, and healthy pancreas using immunohistochemistry and immunofluorescence. The authors confirmed that CD44 and CD133 expression is present in normal and inflammatory pancreatic tumors. However, the localization of these markers evaluated by immunofluorescence was different in the pancreatic tissue. In normal pancreas, CD44 and CD133 were located in centroacinar region with CD44 being present in the basolateral membrane and CD133 apically. Interestingly, CD44 and CD133 did not colocalize on the membranes of normal and inflamed pancreatic cells. Similar results were shown in PDAC. No quantitative analysis was done. Additionally, the authors showed that CD44 expression was higher in patients with advanced diseases represented by lymph node involvement (N1) compared to that in individuals without lymph node metastases (N0). The expression of CD44, however, did not correspond to overall survival [25]. In our study, immunohistochemical analysis allowed for detecting only membranous and cytoplasmatic expression of examined biomarkers. The presence of CD44 and CD133 in both PDAC and CP corresponds to our results. We demonstrated higher expression of CD44 in PDAC compared to $\mathrm{CP}$ and no significant differences in the expression of CD133 in PDAC and CP. In contrast to the study of Immervoll et al., our data shows the lower immunoreactivity of CD44 in advanced PDAC $(\mathrm{T} 3, \mathrm{~N} 1)$ in comparison to locally advanced disease (T1, T2, and N0). As shown by many authors, the correlation of CD44 expression and the prognosis in various cancers is not uniform [26-28]. As the role of CD44 in carcinogenesis is not fully elucidated, its prognostic value still cannot be established.

Palagani et al. measured CD44 levels in serum of patients undergoing chemotherapy in PDAC, colon cancer, and gastric cancer. The authors reported a decrease in CD44 levels in patients who responded to treatment [29]. Here, we demonstrated that CD44 expression is higher in patients with pancreatic cancer compared to those with chronic pancreatitis. However, the local advancement of PDAC (T3) and lymph node involvement (N1) inversely correlated with CD44 expression. As CD44 expression has been proved in most malignancies, the exact role of this molecule in carcinogenesis and tumour biology is still unclear. Our results suggest that loss of CD44 expression in advanced PDAC might be a negative prognostic factor in this disease. The molecular background of this phenomenon still needs to be elucidated. Additionally, further studies requiring different analytical methods, such as immunofluorescence, quantitative PCR, or flow cytometry analyses, are needed to confirm this data.

In our study, we have also found similar CD133 immunoreactivity in PDAC and CP patients. Many previous studies confirmed expression of CD133 in majority types of malignant neoplasms, including PDAC [8, 10, 15]. Vizio et al. evaluated the expression of CSC in tissue samples of normal pancreas and PDAC using immunohistochemistry. The authors showed that the immunoactivity of CD133 did not differ between both groups of analyzed patients. Furthermore, CD133 expression did not correlate with tumor stage [30]. Interestingly, similar results were shown in murine model of PDAC. Dosch et al. examined CSC phenotypes in PDAC using lucipherase tags and reported that CD133and CD24-positive cells were present in both tumorigenic and nontumorigenic cells [31]. Our findings correspond to these observations. As CD133 is an established marker of CSC in PDAC, its expression might not represent disease activity and does not allow for early detection of this neoplasm. One might speculate that the presence of this biomarker in normal or inflamed pancreatic tissue could represent the cells of possible CSC potential, which may be prone to undergo malignant transformation.

Our study also demonstrated that the expression of CD24 was higher in PDAC than in CP. A high expression of this marker was observed in various neoplasms by multiple authors. The meta-analysis presented by Lee et al. analyzed 


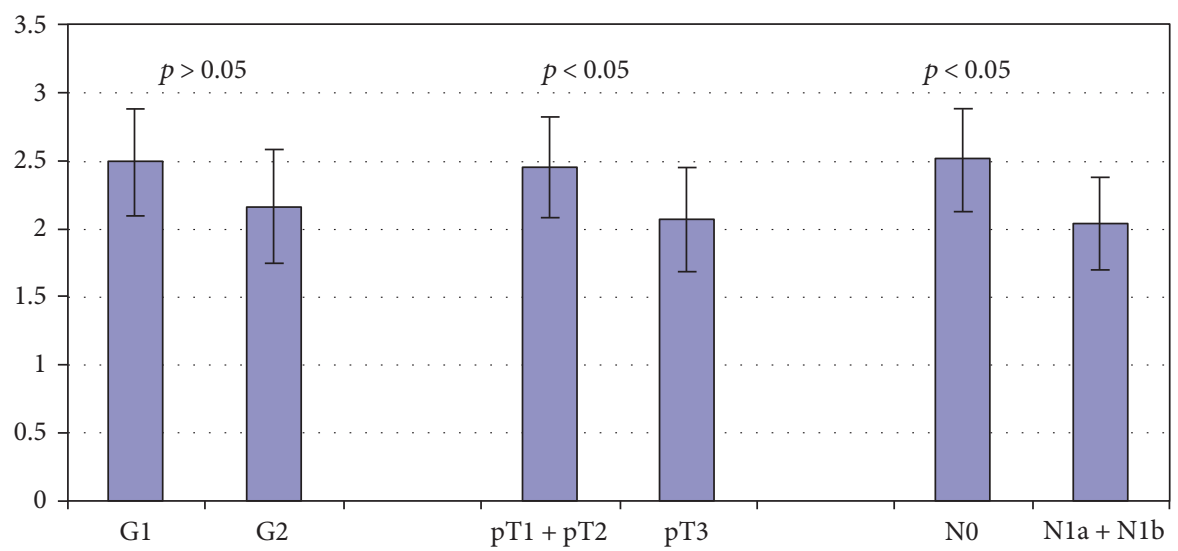

Figure 8: CD44 staining intensity in PDAC depending on tumor differentiation and stage.

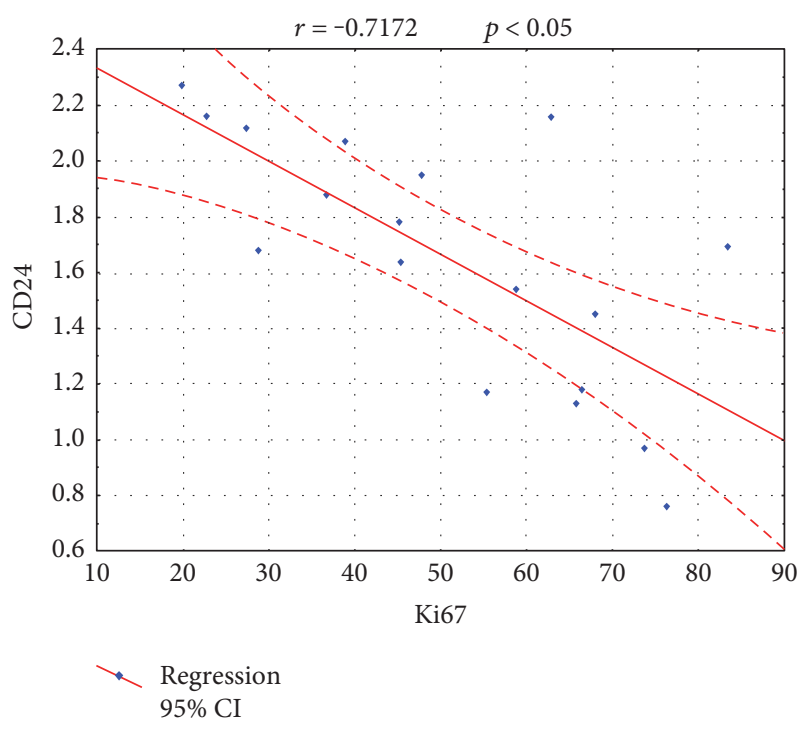

FIGURE 9: The negative correlation between CD24 staining intensity and Ki67 LI in PDAC.

twenty-eight studies on CD24 expression in the breast, female genital tract, gastrointestinal tract, biliary tract, pancreas, urinary system, prostate, and skin carcinomas. Overall, CD24 was more frequently overexpressed in malignant than in benign lesions found within these organs and was significantly associated with lymph node infiltration, advanced clinical stages, and shortened overall survival [32]. Another interesting study on CD24 role in carcinogenesis of PDAC reported increased expression of this marker in preinvasive lesions-pancreatic intraepithelial neoplasia (PanIN). CD24 immunoreactivity was present in $59 \%$ specimen of PanIN I, $87 \%$ of PanIN II, and $100 \%$ of PanIN III [33].

In our study, the values of Ki67 LI in PDAC were higher than those in CP. The expression of Ki67 characterizes the intensity of cell proliferation and is known as a parameter of tumor aggressiveness and prognosis. High Ki67 expression was observed in PDAC by many authors [34-36].

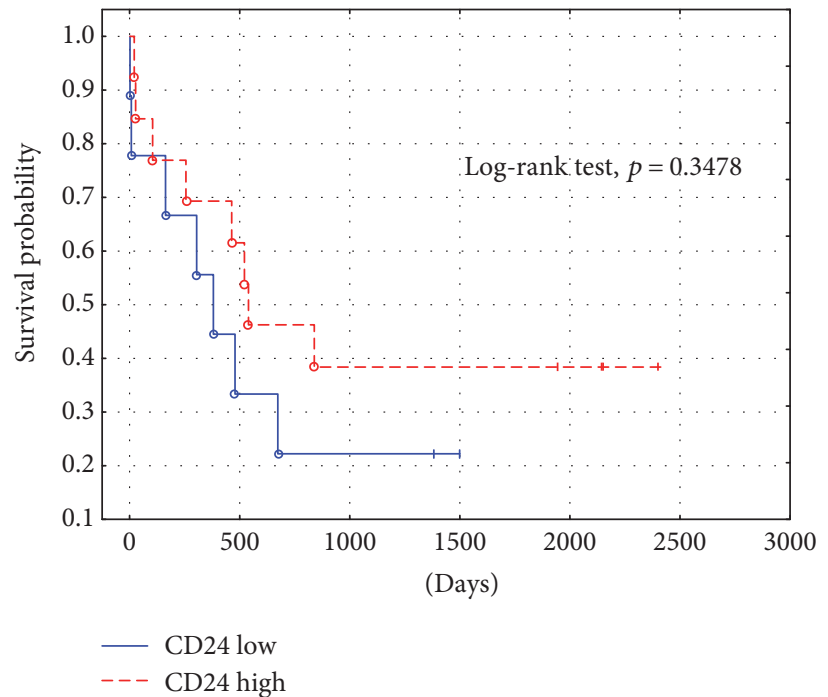

Figure 10: PDAC survival probability depending on CD24 staining intensity.

Since CD24 staining intensity was decreasing with higher cell proliferation, it may be possibly considered as an early PDAC marker. Lack of correlation between the expression of CD44 and CD133 with Ki67 immunoreactivity in PDAC might correspond to advanced cell proliferation in all examined specimen including cells without CSC properties. Thus, this finding does not rule out the role of examined CSC biomarkers in the development of PDAC. As to our knowledge, there were no large studies revealing the correlation of CSC markers and Ki67 proliferation index.

Our study showed no correlation between PDAC patients' survival and the expression of CD24, CD44, and CD133. A meta-analysis by $\mathrm{Li}$ et al. which analyzed the relation of CD133 expression and survival in PDAC performed on the group of 908 patients showed shorter survival of individuals with high expression of CD133 comparing to those with low expression of this biomarker 


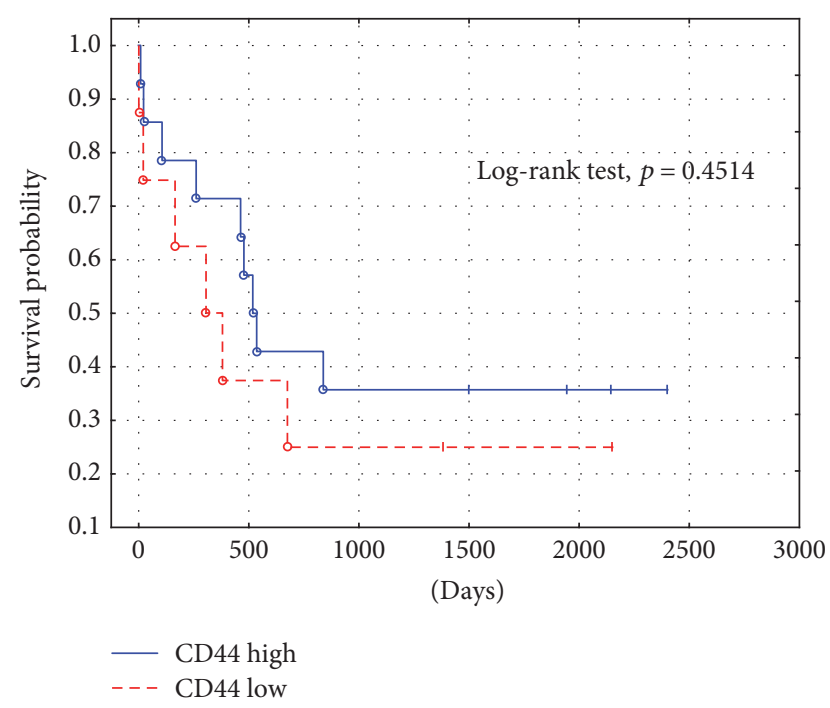

FIGURE 11: PDAC survival probability depending on CD44 staining intensity.

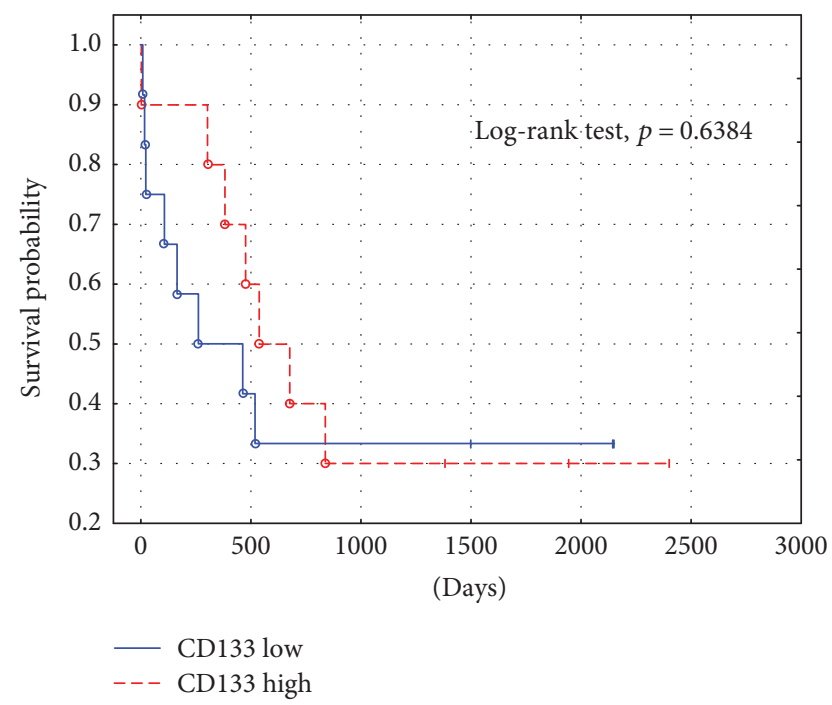

FIGURE 12: PDAC survival probability depending on CD133 staining intensity.

[37]. Similarly, high expression of CD24 in PDAC correlated with poor prognosis in the study of Lee et al. performed on the group of 67 PDAC with resected tumor [38]. Additionally, an analysis on 96 PDAC patients by Hou et al. showed shorter survival in individuals who presented coexpression of CD44 and CD133 [39]. All presented studies were based only on Asian population, whereas similar data from Western countries is limited. Moreover, all analyses did not provide data on supplemental chemo- or radiotherapy data which also influenced the prognosis of PDAC patients. However, our study analysed a smaller number of patients; it included individuals who did not receive systemic treatment which certainly decreased overall survival rates.

\section{Conclusions}

CD24 and CD44 are upregulated in human pancreatic cancer compared to chronic pancreatitis and may be related to the development of pancreatic cancer. Loss of CD44 expression in advanced stages of PDAC could indicate unfavorable prognosis and may represent a negative prognostic factor. More detailed studies on the role of CD133 cells in both diseases is necessary.

\section{Conflicts of Interest}

The authors declare that they have no conflicts of interest.

\section{Acknowledgments}

The authors received a grant from a Medical University of Lodz grant for young scientists (502-03/1-002-01/502-14045). They also received funds from the Medical University of Lodz statutory funds (503/1-002-01/503-11-003). The authors also thank the Polish Society for the Prevention of Digestive Tract Neoplasms.

\section{References}

[1] L. Rahib, B. D. Smith, R. Aizenberg, A. B. Rosenzweig, J. M. Fleshman, and L. M. Matrisian, "Projecting cancer incidence and deaths to 2030: the unexpected burden of thyroid, liver, and pancreas cancers in the United States," Cancer Research, vol. 74, no. 11, pp. 2913-2921, 2014.

[2] C. F. Kim, E. L. Jackson, A. E. Woolfenden et al., "Identification of bronchioalveolar stem cells in normal lung and lung cancer," Cell, vol. 121, no. 6, pp. 823-835, 2005.

[3] C. A. O'Brien, A. Pollett, S. Gallinger, and J. E. Dick, "A human colon cancer cell capable of initiating tumour growth in immunodeficient mice," Nature, vol. 445, no. 7123, pp. 106110, 2007.

[4] A. T. Collins, P. A. Berry, C. Hyde, M. J. Stower, and N. J. Maitland, "Prospective identification of tumorigenic prostate cancer stem cells," Cancer Research, vol. 65, no. 23, pp. 10946-10951, 2005.

[5] P. P. Szotek, R. Pieretti-Vanmarcke, P. T. Masiakos et al., "Ovarian cancer side population defines cells with stem celllike characteristics and Mullerian inhibiting substance responsiveness," Proceedings of the National Academy of Sciences of the United States of America, vol. 103, no. 30, pp. 1115411159, 2006.

[6] C. Li, D. G. Heidt, P. Dalerba et al., "Identification of pancreatic cancer stem cells," Cancer Research, vol. 67, no. 3, pp. 1030-1037, 2007.

[7] P. C. Hermann, S. L. Huber, T. Herrler et al., "Distinct populations of cancer stem cells determine tumor growth and metastatic activity in human pancreatic cancer," Cell Stem Cell, vol. 1, no. 3, pp. 313-323, 2007.

[8] Z. Li, "CD133: a stem cell biomarker and beyond," Experimental Hematology \& Oncology, vol. 2, no. 1, p. 17, 2013.

[9] X. Fang, P. Zheng, J. Tang, and Y. Liu, "CD24: from A to Z," Cellular \& Molecular Immunology, vol. 7, no. 2, pp. 100-103, 2010.

[10] J. Skoda, M. Hermanova, T. Loja et al., "Co-expression of cancer stem cell markers corresponds to a pro-tumorigenic 
expression profile in pancreatic adenocarcinoma," PLoS One, vol. 11, no. 7, article e0159255, 2016.

[11] X. P. Li, X. W. Zhang, L. Z. Zheng, and W. J. Guo, "Expression of CD44 in pancreatic cancer and its significance," International Journal of Clinical and Experimental Pathology, vol. 8, no. 6, pp. 6724-6731, 2015.

[12] E. V. Abel, E. J. Kim, J. Wu et al., "The Notch pathway is important in maintaining the cancer stem cell population in pancreatic cancer," PLoS One, vol. 9, no. 3, article e91983, 2014.

[13] M. Rodova, J. Fu, D. N. Watkins, R. K. Srivastava, and S. Shankar, "Sonic hedgehog signaling inhibition provides opportunities for targeted therapy by sulforaphane in regulating pancreatic cancer stem cell self-renewal," PLoS One, vol. 7, no. 9, article e46083, 2012.

[14] S. Matsubara, Q. Ding, Y. Miyazaki, T. Kuwahata, K. Tsukasa, and S. Takao, "mTOR plays critical roles in pancreatic cancer stem cells through specific and stemness-related functions," Scientific Reports, vol. 3, p. 3230, 2013.

[15] A. Van den Broeck, H. Vankelecom, W. Van Delm et al., "Human pancreatic cancer contains a side population expressing cancer stem cell-associated and prognostic genes," PLoS One, vol. 8, no. 9, article e73968, 2013.

[16] S. P. Hong, J. Wen, S. Bang, S. Park, and S. Y. Song, "CD44positive cells are responsible for gemcitabine resistance in pancreatic cancer cells," International Journal of Cancer, vol. 125, no. 10, pp. 2323-2331, 2009.

[17] S. Asuthkar, V. Stepanova, T. Lebedeva et al., "Multifunctional roles of urokinase plasminogen activator (uPA) in cancer stemness and chemoresistance of pancreatic cancer," Molecular Biology of the Cell, vol. 24, no. 17, pp. 2620-2632, 2013.

[18] E. Lonardo, M. Cioffi, P. Sancho et al., "Metformin targets the metabolic Achilles heel of human pancreatic cancer stem cells," PLoS One, vol. 8, no. 10, article e76518, 2013.

[19] V. Rausch, L. Liu, G. Kallifatidis et al., "Synergistic activity of sorafenib and sulforaphane abolishes pancreatic cancer stem cell characteristics," Cancer Research, vol. 70, no. 12, pp. 5004-5013, 2010.

[20] C. Li, J. J. Wu, M. Hynes et al., "c-Met is a marker of pancreatic cancer stem cells and therapeutic target," Gastroenterology, vol. 141, no. 6, pp. 2218-2227, 2011.

[21] S. Midha, S. Chawla, and P. K. Garg, "Modifiable and nonmodifiable risk factors for pancreatic cancer: a review," Cancer Letters, vol. 381, no. 1, pp. 269-277, 2016.

[22] A. Gasiorowska, R. Talar-Wojnarowska, A. Kaczka, A. Borkowska, L. Czupryniak, and E. Małecka-Panas, "Subclinical inflammation and endothelial dysfunction in patients with chronic pancreatitis and newly diagnosed pancreatic cancer," Digestive Diseases and Sciences, vol. 61, no. 4, pp. 1121-1129, 2016.

[23] A. Kozak, R. Talar-Wojnarowska, A. Kaczka et al., "Utility of different serum fibrosis markers in diagnosing patients with chronic pancreatitis and pancreatic adenocarcinoma," World Journal of Gastrointestinal Oncology, vol. 8, no. 8, pp. 635$641,2016$.

[24] C. Kordes, I. Sawitza, S. Götze, D. Herebian, and D. Häussinger, "Stellate cells are mesenchymal stem cells," European Journal of Medical Research, vol. 19, Supplement 1, p. S6, 2014.

[25] H. Immervoll, D. Hoem, O. J. Steffensen, H. Miletic, and A. Molven, "Visualization of CD44 and CD133 in normal pancreas and pancreatic ductal adenocarcinomas: non- overlapping membrane expression in cell populations positive for both markers," The Journal of Histochemistry and Cytochemistry, vol. 59, no. 4, pp. 441-455, 2011.

[26] S. Sato, M. Miyauchi, T. Takekoshi et al., "Reduced expression of CD44 variant 9 is related to lymph node metastasis and poor survival in squamous cell carcinoma of tongue," Oral Oncology, vol. 36, no. 6, pp. 545-549, 2000.

[27] J. W. Huh, H. R. Kim, Y. J. Kim et al., "Expression of standard CD44 in human colorectal carcinoma: association with prognosis," Pathology International, vol. 59, no. 4, pp. 241-246, 2009.

[28] M. H. Jang, H. J. Kang, K. S. Jang, S. S. Paik, and W. S. Kim, "Clinicopathological analysis of CD44 and CD24 expression in invasive breast cancer," Oncology Letters, vol. 12, no. 4, pp. 2728-2733, 2016.

[29] V. Palagani, M. El Khatib, T. Krech, M. P. Manns, N. P. Malek, and R. R. Plentz, "Decrease of CD44-positive cells correlates with tumor response to chemotherapy in patients with gastrointestinal cancer," Anticancer Research, vol. 32, no. 5, pp. 1747-1755, 2012.

[30] B. Vizio, F. A. Mauri, A. Prati et al., "Comparative evaluation of cancer stem cell markers in normal pancreas and pancreatic ductal adenocarcinoma," Oncology Reports, vol. 27, no. 1, pp. 69-76, 2012.

[31] J. S. Dosch, E. K. Ziemke, A. Shettigar, A. Rehemtulla, and J. S. Sebolt-Leopold, "Cancer stem cell marker phenotypes are reversible and functionally homogeneous in a preclinical model of pancreatic cancer," Cancer Research, vol. 75, no. 21, pp. 4582-4592, 2015.

[32] J. H. Lee, S. H. Kim, E. S. Lee, and Y. S. Kim, "CD24 overexpression in cancer development and progression: a meta-analysis,” Oncology Reports, vol. 22, no. 5, pp. 1149-1156, 2009.

[33] X. Pei, J. Zhu, R. Yang et al., "CD90 and CD24 co-expression is associated with pancreatic intraepithelial Neoplasias," PLoS One, vol. 11, no. 6, article e0158021, 2016.

[34] F. Stipa, G. Lucandri, M. R. Limiti et al., "Angiogenesis as a prognostic indicator in pancreatic ductal adenocarcinoma," Anticancer Research, vol. 22, no. 1A, pp. 445-449, 2002.

[35] C. Schlüter, M. Duchrow, C. Wohlenberg et al., "The cell proliferation-associated antigen of antibody Ki-67: a very large, ubiquitous nuclear protein with numerous repeated elements, representing a new kind of cell cycle-maintaining protein," The Journal of Cell Biology, vol. 123, no. 3, pp. 513-522, 1993.

[36] M. Ammendola, R. Sacco, I. Marech et al., "Microvascular density and endothelial area correlate with $\mathrm{Ki}-67$ proliferative index in surgically-treated pancreatic ductal adenocarcinoma patients," Oncology Letters, vol. 10, no. 2, pp. 967-971, 2015.

[37] X. Li, H. Zhao, J. Gu, and L. Zheng, "Prognostic value of cancer stem cell marker CD133 expression in pancreatic ductal adenocarcinoma (PDAC): a systematic review and meta-analysis," International Journal of Clinical and Experimental Pathology, vol. 8, no. 10, pp. 12084-12092, 2015.

[38] S. H. Lee, H. Kim, J. H. Hwang et al., "CD24 and S100A4 expression in resectable pancreatic cancers with earlier disease recurrence and poor survival," Pancreas, vol. 43, no. 3, pp. 380-388, 2014.

[39] Y. C. Hou, Y. J. Chao, H. L. Tung, H. C. Wang, and Y. S. Shan, "Coexpression of CD44-positive/CD133-positive cancer stem cells and CD204-positive tumor-associated macrophages is a predictor of survival in pancreatic ductal adenocarcinoma," Cancer, vol. 120, no. 17, pp. 2766-2777, 2014. 


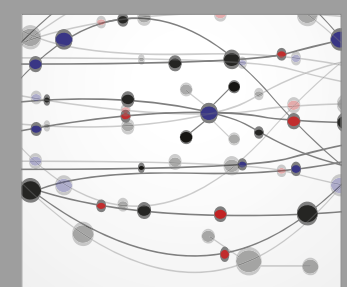

The Scientific World Journal
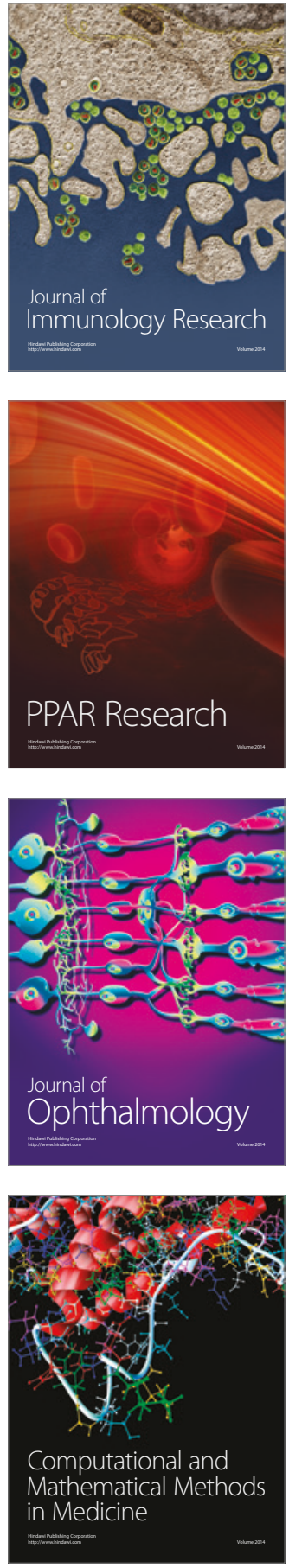

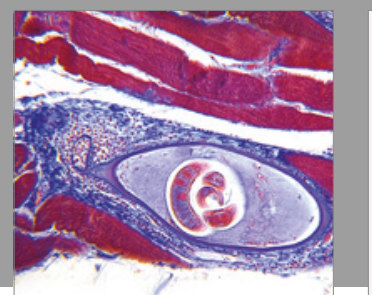

Gastroenterology Research and Practice
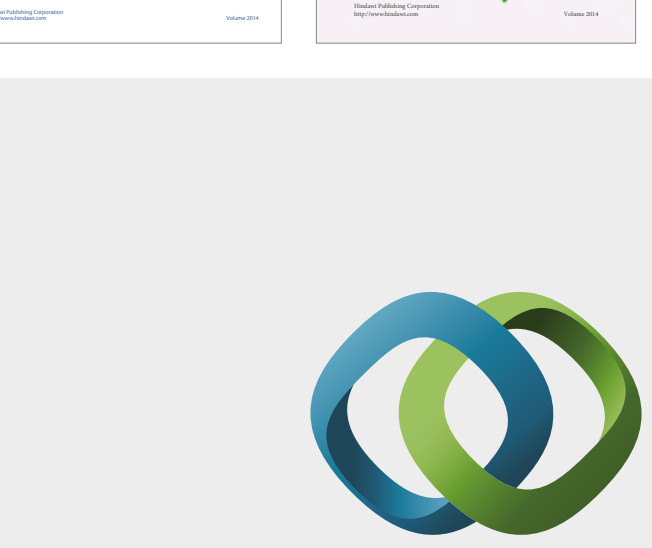

\section{Hindawi}

Submit your manuscripts at

https://www.hindawi.com
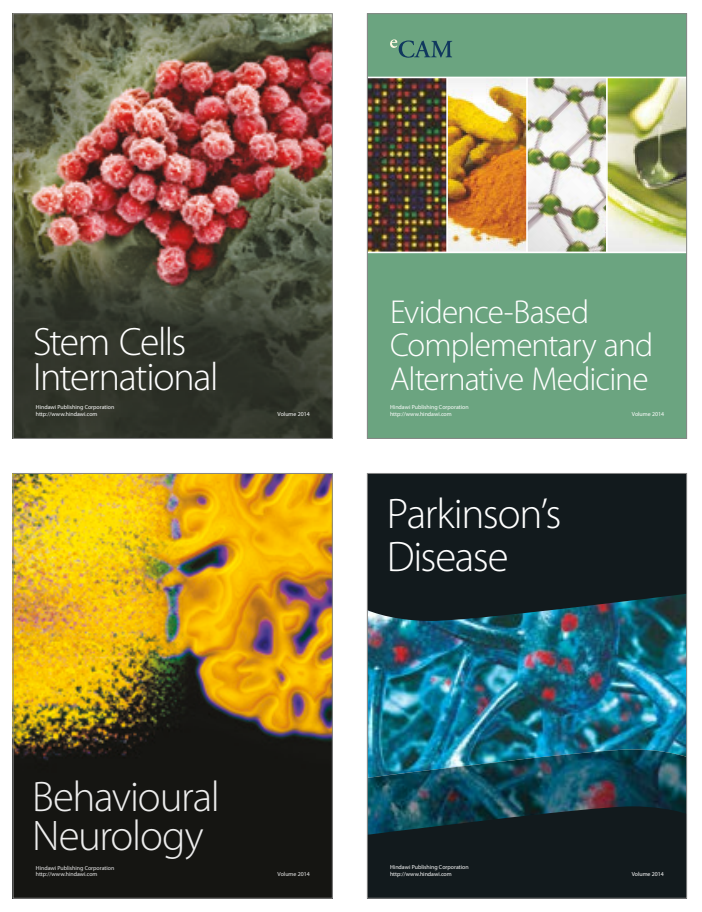
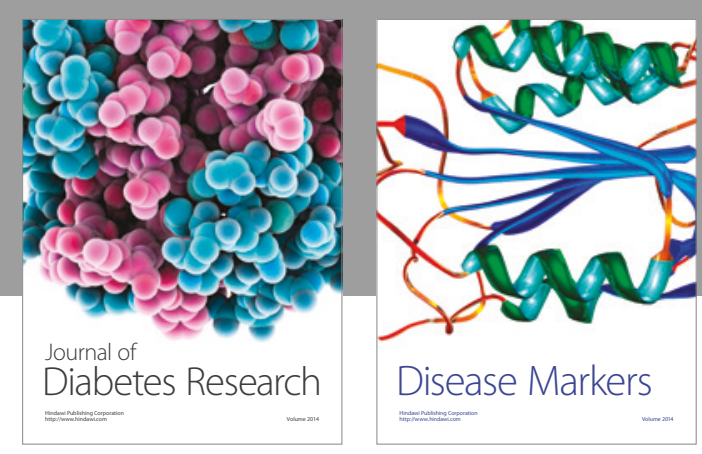

Disease Markers
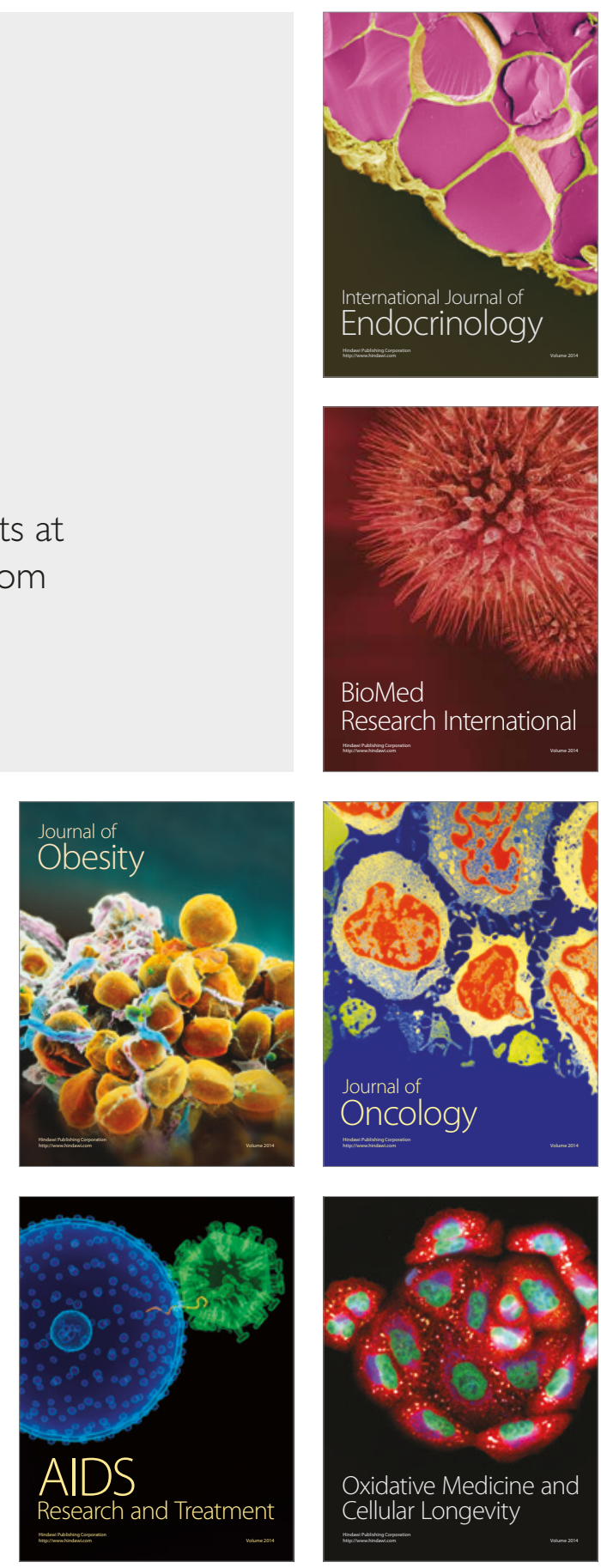